\title{
Deflating Truth about Taste
}

Filippo Ferrari (University of Bonn) \& Sebastiano Moruzzi (University of Bologna)

\section{Introduction}

In Truth and Objectivity, Crispin Wright argues that because truth is a distinctively normative property, it cannot be as metaphysically insubstantive as deflationists claim. ${ }^{1}$ This argument has been taken, together with the scope problem, ${ }^{2}$ as one of the main motivations for alethic pluralism. ${ }^{3}$ We offer a reconstruction of Wright's Inflationary Argument (henceforth IA) aimed at highlighting what are the steps required to establish its inflationary conclusion. We argue that if a certain metaphysical and epistemological view of a given subject matter is accepted, a local counterexample to IA can be constructed.

We focus on the domain of basic taste and we develop two variants of a subjectivist and relativist metaphysics and epistemology that seems palatable in that domain. Although we undertake no commitment to this being the right metaphysical cum epistemological package for basic taste, we contend that if the metaphysics and the epistemology of basic taste are understood along these lines, they call for a truth property whose nature is not distinctively normative-contra what IA predicts.

This result shows that the success of IA requires certain substantial metaphysical and epistemological principles and that, consequently, a proper assessment of IA cannot avoid taking a stance on the metaphysics and the epistemology of the domain where it is claimed to be successful. Although we conjecture that IA might succeed in other domains, in this paper we don't take a stand on this issue. We conclude by briefly discussing the significance of this result for the debate on alethic pluralism.

\section{Wright's Inflationary Argument}

IA targets a family of deflationary conceptions of truth, the most representative of which is Horwich's Minimalism (Horwich 1998). Minimalism amounts to the conjunction of the minimal theory - the propositions expressing the non-paradoxical instances of the equivalence schema: ES $\quad<p>$ is true if and only if $p$,

\footnotetext{
1 Wright (1992, Ch.1).

2 See, e.g., Lynch 2009: 4.

3 See, e.g., Wright 1998.
} 
and the minimalist conception which illustrates the conceptual and metaphysical commitments of deflationism. Among other commitments, what is most characteristic of this view is the thesis that 'true' denotes a genuine property, albeit a metaphysically insubstantive one (INSUBSTANTIALITY) i.e. a property which plays no explanatory role. ${ }^{4}$

In what follows, we offer our own reconstruction of Wright's IA and we take it to show three things. ${ }^{5}$ First, that truth and justification mark distinct norms of belief. Second, that what is responsible for this normative divergence is a difference in intrinsic features of the natures of truth and justification. Third, that in virtue of its normative nature truth plays a non-trivial explanatory role vis-à-vis some core phenomena of enquiry. The conclusion is that truth cannot be an insubstantive property. We offer our reconstruction of IA in seven steps.

The first step —Normativity — assumes that both truth and justification function as norms of belief in the minimal sense that they both provide criteria for the correctness of the formation and retraction of beliefs.

The second step-Normative Coincidence-claims that truth and justification coincide in positive normative force: any reason to regard a proposition as justified is, naturally, a reason to endorse it as belief, and, conversely, any reason to endorse a proposition as belief is, by ES, also a reason to regard the proposition as true. Thus, any reason to regard a proposition as justified is a reason to regard it as true, and conversely (Wright 1992: 18).

The third step-Normative Divergence-establishes that truth and justification potentially diverge in extension: while 'true' commutes with negation, 'justified' does not. The following negation equivalence holds for truth:

$\mathrm{NE} \quad<\mathrm{p}>$ is not true if and only if $<$ not $\mathrm{p}>$ is true. ${ }^{6}$

but it fails for justification. NE-J fails from the left-to-right direction:

NE-J $\quad<\mathrm{p}>$ is not justified if and only if $<$ not $\mathrm{p}>$ is justified.

The failure of NE-J depends on the availability of a notion of justification that admits of neutral states of information:

\footnotetext{
4 See Wyatt (2016) and Ferrari 2018.

${ }^{5}$ IA has been discussed by Rumfitt (1995), Tennant (1995), (1998) Price (1998), Edwards (1997), (1999), Horwich (1998), Miller (2001) and Lynch (2006).

${ }^{6}$ Proof:

1. $<\mathrm{p}>$ is true if, and only if, $\mathrm{p}$

(equivalence schema)

2. $\langle\mathrm{p}>$ is not true if, and only if, not $\mathrm{p} \quad(1$, contraposition)

3. $<$ not $\mathrm{p}>$ is true if, and only if, not $\mathrm{p} \quad(1$, substitution of ' $\mathrm{p}$ ' with 'not $\mathrm{p}$ ')

4. $<\mathrm{p}>$ is not true if, and only if, $<$ not $\mathrm{p}>$ is true $(2,3$, transitivity). 
(Epistemic Neutrality) a subject $S$ is in an epistemically neutral situation with respect to her informational state $i$ and to a proposition $<\mathrm{p}>$ just in case $i$ provides $\mathrm{S}$ with neither a justification for $<\mathrm{p}>$ nor a justification for $<$ not-p $>$.

Thus, the following might happen: while, for any proposition $<\mathrm{p}\rangle$, either $\langle$ p $>$ or $\langle$ not-p $>$ falls within the extension of 'true', this is not the case for 'justified'-relative to an epistemically neutral state of information both $\langle\mathrm{p}\rangle$ and $\langle$ not-p $>$ fall outside the extension of 'justified'. As a consequence of this potential extensional divergence we have that truth and justification might give conflicting normative predictions. A potential divergence in normative role between truth and justification is thus ensured.

The fourth step-Generality — consists in the observation that this argumentative pattern can be replicated for any other property which is positively normatively coincident with truth-e.g. knowledge. This is because these notions are at least partly epistemic in nature in that they make reference to the subject's informational state at the relevant time of belief formation when the coincidence in positive normative force is established.

The fifth step-Intrinsicality-purports to establish that truth's normative function is a sui generis feature that belongs to its nature and it's not derived from that of other notions which play a normative role, such as justification or knowledge. This is taken to be a corollary of the previous steps - truth has to be a distinctively normative notion given that its normativity is distinct from and cannot be reduced to that of either justification or knowledge.

The sixth step-Grounding - maintains that in virtue of its distinctive normative nature truth plays a crucial role in the explanation of the normative significance of an array of enquiry-related phenomena. ${ }^{7}$ The notion of explanation in play here can be understood in terms of metaphysical grounding. As Kit Fine puts it, the notion of grounding provides a "distinctive kind of metaphysical explanation, in which explanans and explanandum are connected, not through some sort of causal mechanism, but through some constitutive form of determination.” (Fine 2012: 37). The thought is that truth's normative nature grounds, at least in part, the normative significance of an array of enquiry-related phenomena-e.g. the possibility and distinctive normative significance of disagreement. ${ }^{8}$

The seventh step-Inflation - concludes that in virtue of its grounding role truth's nature cannot be purely deflationary. This is because if truth takes part to the metaphysical determination

\footnotetext{
7 Wright's original argument takes no issue with grounding and intrinsicality - these are steps that we've introduced to make clearer the connection between truth's being a normative property and its inflated nature.

8 See Price (1998).
} 
of an array of enquiry-related normative phenomena and it does so in virtue of some of its intrinsic features, then there must be something about truth's nature which is responsible for such a determination. This rejects the claim that truth is a metaphysically and explanatorily inert property. As a consequence, a certain — not yet determined in magnitude-degree of inflation of truth's nature has to be granted. INSUBSTANTIALITY is thus false.

There are various strategies to respond to IA, depending on which of the aforementioned steps are contested. One might contest Intrinsicality by claiming that IA falls short of establishing that truth is an intrinsically normative notion. This is the strategy that Horwich in some recent work has defended (see Horwich 2018 and Ferrari 2018). Or one might reject Grounding by rejecting the explanatory link between truth's normative nature and the normative significance of enquiry-related phenomena (Horwich 1998, Wrenn 2015:122).

In this paper, we won't take issue with the soundness of Intrinsicality and Grounding. Our target is Normative Divergence. We will show how this step can be blocked in the domain of basic taste by outlining a metaphysics and epistemology of basic taste that has the effect of neutralising Normative Divergence.

\section{Metaphysics and Epistemology of Basic Taste}

There are two main components to our metaphysics of taste. ${ }^{9}$ The first is metaphysical subjectivism, the thesis that the instantiation of basic taste properties—e.g. being tasty-entirely depends on the taste sensibility of the beholder-i.e. her gustatory responses (GRs). The second component is metaphysical relativism, the thesis that different beholders with different taste sensibilities give rise to different facts about what is (and is not) tasty. What taste facts are there is entirely determined by the set of gustatory responses of a given subject. Varying the subject or changing the set of gustatory responses of a given subject engenders a change in the world of taste. As a consequence, the extension of a basic taste property is relative to the world of taste of a subject. Reality is thus composed by, at least, two sorts of facts: objective facts—e.g. facts about the physical composition of objects, whose occurrence is totally independent of any subject's perspective-and perspectival facts - e.g. taste facts-whose occurrence fully depends on the gustatory perspective of a subject. ${ }^{10}$

\footnotetext{
${ }^{9}$ In the following we do not take a stand on the semantics of taste predicates on which there is a rich and growing literature. As a methodological principle, we want to keep separate issues of metaphysics from issues on the semantics on natural languages.

10 The kind of metaphysical relativism we adopt is closely related to Fine's external relativism (Fine 2005: 278-284). For alternative versions of metaphysical relativism, see, for instance, Einheuser (2008), Jackson (2016), Rovane (2013), and Spencer (2016).
} 
These two theses mutually support each other. Metaphysical relativism is safeguard to metaphysical subjectivism from contradictory states of affairs: given two subjects with different gustatory responses, a basic taste property can consistently be and not be instantiated in the same object because it is always relative to a subject whether the object has or fails to have that property. ${ }^{11}$ To exemplify: Jülide's gustatory responses determine that sushi is tasty, whereas Elias's gustatory responses determine that sushi is not tasty. However, no glutty facts would follow since whether or not tastiness is instantiated is a matter relative to the subject: that sushi is tasty holds relative to Jülide's perspective, whereas the contradictory proposition holds relative to Elias's.

Moreover, metaphysical subjectivism backs metaphysical relativism up since it provides the source of the kind of relativity predicted by metaphysical relativism. More specifically, what metaphysical subjectivism says is that the subject's gustatory responses shape the perspective of the subject on the world of taste. Different perspectives involve different true propositions about taste- and thus different taste facts.

Metaphysical subjectivism and relativism amount to a relativized form of response dependence (RRD). Focusing on the properties of tastiness and distastefulness as paradigmatic cases for positive and negative gustatory evaluations, RRD makes the determination of properties of tastiness and distastefulness relative to the response of a subject: the very same piece of sushi can be tasty because Jülide has a pleasant reaction in tasting it and, at the same time, it can be distasteful because Elias has an unpleasant reaction in tasting it. If we define a fact as the possession of a property by an object at a time, we have that the fact that sushi is tasty belongs to the world of taste of Jülide and the fact that sushi is distasteful belongs to the world of taste of Elias. In this sense, RRD is our principle of determination of taste-reality. We take taste reality to include, among other things, taste facts such as that a certain piece of sushi $\mathbf{s}$ is tasty simpliciter - in analogy with the terminology of tensed facts introduced in the temporalist debate, we call them tasted facts - and not as composed only of relational facts such as the fact that $\mathbf{s}$ is tasty for Elias. We will provide two different formulations of RRD: one which is compatible with classical semantics ( $(\mathbb{4})$ and the other which is in line with a gappy semantics $(\$ 5)$.

This metaphysics can be paired with an epistemology of basic taste characterised by two key elements: the primacy of experience thesis (PE), and the thesis of positive accessibility of gustatory experience (PAGE). According to PE the justification for a taste proposition consists entirely in the subject's

\footnotetext{
11 What are the identity criteria of food is a rather complex issue and we have no illuminating account to offer on this. As far as we know, the current philosophical literature on basic taste is pretty much silent on this crucial issue-see, e.g., Borghini (2012).
} 
gustatory responses to her experience. Thus, the only source of justification for a taste proposition is first-person gustatory experience. ${ }^{12}$

PAGE is the thesis that since subjects have a privileged access to their gustatory experience, a subject always has epistemic access to the information relative to her gustatory experiences. This means that if a subject has a gustatory experience with a specific quality (i.e. either positive, negative, or neutral) she thereby has propositional justification to believe that she had such an experience with that quality. ${ }^{13}$

If we endorse the metaphysical and epistemological package just outlined, two strategiesone classical and one non-classical—can be developed for blocking the inflationary argument by providing a counterexample to Normative Divergence.

But before turning to this task, let us briefly mention two ways in which we restrict, for the sake of space and simplicity, our discussion of the metaphysical and epistemological package for basic taste. First, we focus exclusively on particular basic taste judgements-i.e. judgements about particular basic taste propositions like <this sushi is delicious $>$. This means that we say nothing concerning generic basic taste judgements-e.g. <sushi is delicious $>$ - and generalisations-e.g. $<$ all food Bottura prepares is delicious $>$. Our motivation for doing so is twofold: on the one hand, generic judgements and generalisations presents additional and difficult concerns that are orthogonal to our project in this paper. On the other hand, such concerns are not specific to our metaphysicscum-epistemology of taste. They point to difficulties that any metaphysical and epistemological picture of basic taste has to face-perhaps to a different degree of intensity, depending on the details of the view. Second, in discussing our view we focus only on one sensory dimension-the purely gustatory one. However, a multi-sensorial approach to taste, which includes, in addition to gustatory elements, also olfactory and visual elements, seems more realistic. We think that such an approach is entirely compatible with the general shape of the metaphysical and epistemological picture just outlined, but for the sake of keeping things simple, in developing the two responses to Wright's IA, we restrict our attention to the purely gustatory dimension.

\footnotetext{
${ }^{12}$ This is in line with the so-called acquaintance principle - see Wollheim (1980) and Robson (2013).

${ }^{13}$ One might object that PAGE falls prey to Williamson's anti-luminosity argument-See Williamson (2000). Our notion of justification for first-person gustatory experiences is non-factive in that it doesn't exclude situations where we have a justification for believing that I have a pleasant experience but where I lack such pleasant experience. So, it's not clear that Williamson's anti-luminosity argument applies to our setting. Moreover, the argument is a reductio of a set of principles: (i) that knowledge of sensations is constrained by margin for error principles (and thus by safety and indiscriminability limitations); (ii) that sensations are luminous, (iii) that it is always possible to construct a soritical-like series for sensations; (iv) that we have a logic that validates transitivity, modus ponens and reductio ad absurdum. Nothing in our picture commits us to the whole package. Thanks to Chase Wrenn and Thomas Grundmann for pressing us on this point.
} 


\section{Classical Response to the Inflationary Argument}

In order to develop a classical-i.e. non-gappy—response to IA we first provide a specific formulation of the relativised form of response dependence that accords with the classical framework (RRD-C) and then we introduce an additional epistemological principle-(NAGE).

According to RRD-C the world of taste of a subject is completely determined. Any specific object of gustatory experience (e.g. a piece of sushi) falls either within the extension of any particular taste property F (e.g. being tasty), or within its anti-extension. It falls within the extension of being tasty if the subject has had a pleasure-like response in relation to it or to some suitably similar object. Otherwise, the sushi in question falls within the anti-extension of the property of being tasty by default. ${ }^{14}$ Thus, relative to a given subject it is always determined whether or not a certain gustatory object (and any suitably similar objects) falls within the extension of a taste property. Let's call homogeneity class of a physical object $\mathrm{x}$ the class of those objects which physical composition varies only slightly from that of $\mathrm{x}$. Though the notion of homogeneity class is vague (since the notion of slight variation itself is vague) we take it to be sufficiently clear for our purposes-it is class of objects centered around a certain physical object $\mathrm{x}$ on the basis on some physical resemblance that $\mathrm{x}$ bears with these objects. We take the relevant notion of (slight) variation to be a relation which is nontransitive, symmetric and reflexive. The response dependence thesis on taste is thus the thesis that a piece of food $\mathrm{x}$ is tasty relatively to subject at a certain time because the latest experience the subject has had with $x$ or any member of its homogeneity class has a certain phenomenological character such that it has engendered a pleasure-like response in the subject. More precisely:

(RRD-C) $\mathrm{x}$ is tasty/distasteful relative to a world of taste $<\mathrm{w}, \mathrm{s}, \mathrm{t} 1>$ if and only if (because) the latest object $\mathrm{y}$ belonging to the homogeneity class of $\mathrm{x}$ has produced at $\mathrm{t} 0(\mathrm{t} 0 \leq \mathrm{t} 1)$ in $w$ a gustatory experience $\mathrm{P}$ with a certain phenomenological quality (e.g. pleasantness or unpleasantness) in $\mathrm{S}$ such that $\mathrm{P}$ engenders, in the absence of any disabling factor, a pleasure/displeasure-like response in the subject at $\mathrm{t} 0$ in $w$.

RRD-C predicts that if a subject has had no gustatory experience of $\mathrm{x}$, nor of any y belonging to $\mathrm{x}$ 's homogeneity class, then $\mathrm{x}$ falls within the anti-extension of every basic taste property-i.e. it is nottasty, not-disgusting etc., relative to the subject. Note however, that this is congruent with the idea

14 A defence of the idea that the negated atomic property applies when there is no reason to think that the (non-negated) property applies can be found in Williamson (1997: 223-6). The thought is that the negation of an atomic predicate is maximally unspecific, in the sense that is does not commit to anything specific about the object to which it applies to. 
of relativised response dependence since an object falling within the anti-extension of a gustatory property (e.g. being tasty) doesn't imply that it falls within the extension of any of its contrary property (e.g. being distasteful). In fact, for an object to fall within the extension of being distasteful relative to a subject, the subject must have had a displeasing response to it or to some other object belonging to the same homogeneity class.

To further develop the classical strategy, we need to introduce an additional epistemological principle which fits RRD-C. This amounts to a form of negative introspection according to which if a subject $\mathrm{S}$ has had no experience whatsoever of a certain gustatory object $\mathrm{x}$, she will have justification to believe that she has had no experience of $\mathrm{x}$ :

(NAGE) Negative Accessibility of Gustatory Experience: If $\mathrm{S}$ has had no gustatory experience of $\mathrm{x}$ at $\mathrm{t}$ and at any earlier time, then $\mathrm{S}$ has propositional justification to believe that she has had no gustatory experience of $\mathrm{x}$ at $\mathrm{t}$ and at any earlier time.

If we conjoin NAGE with PAGE, we obtain a transparency condition: there is always some propositional justification available to the subject to believe whether or not a certain gustatory experience has occurred.

We have now all the ingredients to develop the classical strategy. Given that IA crucially relies on the possibility of neutral states of information, to block the argument it suffices to show that for some propositional domain no neutral state of information can occur. Showing this amounts to show that IA fails locally.

We begin by distinguishing between what arguably are the only two sources of epistemic neutrality: i) phenomenological neutrality, which occurs when a subject has a gustatory experience with a qualitative aspect that engenders a neutral response-neither pleasant nor unpleasant-in the subject; ii) radical neutrality, which occurs when a subject has had no gustatory experience whatsoever of a particular gustatory object. Neither source sustains the kind of epistemic neutrality required by IA in the taste domain.

Let's discuss phenomenological neutrality first. Consider a sample of food $\mathbf{f}$, which is the latest object belonging to $\mathbf{f}^{\prime}$ s homogeneity class which the subject $\mathrm{S}$ has experienced. Now, suppose S's informational state $i$ is such that she has a phenomenologically neutral experience of $\mathbf{f}$. Because the response is not pleasant, via RRD-C, we have that $\mathbf{f}$ lacks the property of tastiness. If we assume that $\mathrm{S}$ has an a priori justification at $i$ for $\mathrm{RRD}-\mathrm{C},{ }^{15}$ via PAGE, $\mathrm{S}$ can introspect her gustatory

${ }^{15}$ If RRD-C is true, we have default a priori propositional justification for it - qua metaphysical truth. 
responses so that to obtain justification at $i$ to believe that she has a qualitatively neutral response, then, via closure of justification under (justifiably believed) logical consequence, we obtain that $S$ has justification at $i$ to believe that $\mathbf{f}$ is not tasty. Thus, if $\mathrm{S}$ is in an informational state $i$ of qualitative neutrality with respect to $\mathbf{f}$, she has justification at $i$ for $<\mathbf{f}$ is not tasty $>$. But having a justification at $i$ for $<\mathbf{f}$ is not tasty $>$ is incompatible with being in a state of epistemic neutrality.

Radical neutrality obtains in cases of absolute lack of gustatory experience of $\mathbf{f}$. Let's assume that $S$ is at $t$ in a state of radical neutrality with respect to $\mathbf{f}$. We have then two sub-cases: either (a) $S$ is in a state of radical neutrality with respect to all the members of $\mathbf{f}$ 's homogeneity class or (b) she is not.

Let's first consider the sub-case (a). If $\mathrm{S}$ is in a state of radical neutrality with respect to all the members of $\mathbf{f}$ 's homogeneity class, then, by RRD-C $\mathbf{f}$ is not tasty relative to $<w, s, t>$. According to NAGE, $\mathrm{S}$ is justified in believing that, at $\mathrm{t}$ and at any earlier time, she has had no gustatory experience of $\mathbf{f}$ if, a fortiori, she has never had any gustatory experience of $\mathbf{f}$ with a qualitative character engendering a pleasure like response in S. Radical neutrality and NAGE thus entail that $S$ has justification at $i$ for $<$ it is not the case that $S$ has had at $t$ or at any earlier time an experience of f with a certain qualitative aspect that engenders, absent disabling factors, a pleasant gustatory response in $\mathrm{S}>$ — call this latter fact $J$-f-Absence. If we apply NAGE to each member of f's homogeneity class, we obtain the analogous of J-f-Absence for each of these objects. Moreover, assuming that propositional justification is closed under conjunction we have that $\mathrm{S}$ has justification at $i$ for $<$ it is not the case that $S$ has had at $t$ or at any earlier time an experience of a member of $\mathbf{f}^{\prime} s$ homogeneity class with a certain qualitative aspect that engenders, absent disabling factors, a pleasant gustatory response in $\mathrm{S}>$. If we suppose further that $\mathrm{S}$ has a an priori justification at $i$ for RRD-C, it follows by closure of propositional justification under (justifiably believed) logical consequence and $\mathbf{J}$-h-f-Absence that $\mathbf{S}$ has a justification at $i$ for $<\mathbf{f}$ is not tasty $>$. But having a justification at $i$ for $<\mathbf{f}$ is not tasty $>$ is incompatible with S's being in a state of epistemic neutrality.

Let us now consider the sub-case (b). There are times earlier than $t$ when $S$ had an experience with a member of $\mathbf{f}$ 's homogeneity class. Take the latest object of $\mathbf{f}$ 's homogeneity class that $\mathrm{S}$ has experienced. There are three cases: the experience was neutral (b1); it was pleasant (b2); or, it was unpleasant (b3). In case (b1) we fall back to the case of phenomenological neutrality and, by same the reasoning as before, $\mathbf{f}$ is not tasty and the subject has a justification for this. In case (b2), RRD$\mathrm{C}$ says that $\mathbf{f}$ is tasty, moreover PAGE says that, at the time of the experience $\mathrm{t} 0, \mathrm{~S}$ has justification for believing that she has had a pleasant experience and that justification is preserved at $\mathrm{t}$-absent defeaters and given, by hypothesis, that between $\mathrm{t} 0$ and $\mathrm{t} \mathrm{S}$ has had no other gustatory experiences 
of the object. So, assuming $\mathrm{S}$ has an a priori justification for RRD-C, by closure $\mathrm{S}$ has a justification for believing that $\mathbf{f}$ is tasty. By an analogous reasoning, case (b3) delivers that $\mathbf{f}$ is not tasty and that $\mathrm{S}$ has a justification for believing that $\mathbf{f}$ is not tasty

In conclusion, since no genuine state of epistemic neutrality can occur in the domain of basic taste, Normative Divergence is blocked. ${ }^{16}$

\section{Non-Classical Response to the Inflationary Argument}

The main reason for dissatisfaction with the classical strategy has to do with NAGE since it implies that a subject is in a position to be justified in believing of a potentially infinite number of gustatory experiences that she has had none of them. A second source of dissatisfaction might be that RRD$\mathrm{C}$ predicts that the anti-extension of a given basic taste property $\mathrm{F}$, relative to a subject $\mathrm{S}$, is always determined even in those cases where $\mathrm{S}$ has had no gustatory experience whatsoever. Intuitively, if we take seriously the idea that the instantiation of a basic taste property $\mathrm{F}$ is determined solely by a subject's gustatory responses to an object of gustatory experience $\mathbf{f}$, it seems natural to say that in those cases where $\mathrm{S}$ has had no gustatory experience whatsoever of $\mathbf{f}$ nor of any other members of $\mathbf{f}$ 's homogeneity class, no basic taste property $\mathrm{F}$ is instantiated in $\mathbf{f}$ relative to the world of taste of $\mathrm{S}$ and thus that, within that world of taste, any proposition ascribing $\mathrm{F}$ to $\mathbf{f}$ would turn out as neither true nor false.

A non-classical strategy can be developed which is immune to the worries targeting the classical strategy. The core idea is that both the extension and the anti-extension of a given basic taste property are determined by the actual gustatory experiences of the subject. An immediate consequence is that in cases where a subject has had no gustatory experience whatsoever of $\mathbf{f}$ nor of any members of $\mathbf{f}$ 's homogeneity class-i.e. cases of radical neutrality $-\mathbf{f}$ falls neither in the extension nor in the anti-extension of any basic taste property. What this amounts to, from a metaphysical point of view, is that taste reality is potentially gappy. This picture requires an alternative formulation of RRD that is compatible with this metaphysical framework. A simple way to do so is to turn RRD into a conditional principle-i.e. to conditionalise the instantiation of a basic taste property upon a subject actual gustatory experience according to the following principle:

\footnotetext{
16 The mere co-extensionality between the truth property and the justification property does not of course entail that the two concepts are indistinguishable: the two concepts can be kept functionally distinct even though the relevant properties happen to be locally extensionally equivalent.
} 
(RRD-NC) If $\mathrm{S}$ has any gustatory experience of $\mathrm{x}$ at $t$ or of some member of its homogeneity class, then $\mathrm{x}$ is tasty/distasteful relative to a world of taste $<\mathrm{w}, \mathrm{s}, \mathrm{t} 1>$ if and only if (because) the latest object $\mathrm{y}$ that $\mathrm{S}$ has experienced and which belongs to x's homogeneity class, has produced at $\mathrm{t} 0$ $(\mathrm{t} 0 \leq \mathrm{t} 1)$ in $w$ a gustatory experience $\mathrm{P}$ with a certain phenomenological quality (e.g. pleasantness or unpleasantness) in $\mathrm{S}$ such that $\mathrm{P}$ engenders, in the absence of any disabling factor, a pleasure/displeasure-like response in $\mathrm{S}$ at $\mathrm{t} 0$ in $w$.

RRD-NC predicts that taste properties have determinate extensions only insofar as the subject has experienced some members of the the homogeneity class of the food—otherwise both the extension and the anti-extension of the property remain undetermined with respect to that food.

How does RRD-NC help with blocking IA? Concerning phenomenological neutrality, the counter-argument works similarly to that developed for the classical strategy. These are cases where the subject has a kind of experience of $\mathbf{f}$ which engenders a neutral response. This means that the latest object belonging to $\mathbf{f}$ 's homogeneity class that $\mathrm{S}$ has experienced falls by default within the anti-extension of all basic taste properties-i.e. it falls within the anti-extensions of being delicious, disgusting, tasty, distasteful, etc. By a reasoning analogous to that developed in $\$ 4$, using PAGE, the subject has propositional justification for believing that all basic taste properties are not instantiated-i.e. she has propositional justification for believing that the object in question is not delicious, not disgusting, not tasty, not distasteful etc.

Concerning radical neutrality, the counter-argument works differently from that of the classical strategy with respect the sub case (b1) - i.e. the case where the subject has had no experience of any of member of $\mathbf{f}^{\prime}$ 's homogeneity class. Since in this framework truth-value gaps are allowed, cases like (b1) are such that, for any basic taste property $\mathrm{P}, \mathbf{f}$ falls neither within the extension nor the anti-extension of $\mathrm{P}$-i.e. $\mathbf{f}$ falls neither within the extensions nor the anti-extensions of all such properties as being delicious, disgusting, tasty, distasteful, etc. Thus, if S has had no gustatory experience of $\mathbf{f}$ nor of any other member of $\mathbf{f}$ 's homogeneity class, any propositions ascribing a basic taste property, as well as its negation, to $\mathbf{f}$ is neither true nor false within the world of taste of $\mathrm{S}$. Given that $\mathrm{S}$ is in a (b1) state of radical neutrality with respect to $\mathrm{f}$, PAGE cannot deliver to $\mathrm{S}$ propositional justification to believe any positive ascription of any basic taste property $\mathrm{P}$ to $\mathbf{f}$. Moreover, because we don't have NAGE, there's no way for $\mathrm{S}$ to acquire propositional justification to believe any negative ascription of any $\mathrm{P}$ to $\mathbf{f}-\mathrm{e} . \mathrm{g}$. that $\mathbf{f}$ is not tasty, not distasteful, etc.

Thus, in the non-classical framework cases of epistemic neutrality sourced in states of radical neutrality might happen. However, this has no consequences with respect to the issue of the 
potential extensional divergence between truth and justification. In fact, those propositions to which the subject is in a state of epistemic neutrality because of radical neutrality of the (b1) sort, are those that fall neither within the extension nor the anti-extension of truth. Thus, even in these cases there is a perfect alignment between truth and justification.

Before moving on, there are two worries concerning the non-classical strategy that are worth discussing. Firstly, one might worry that working within a framework that admits of truth-value gaps neutralises IA right from the get-go since the negation equivalence is invalidated:

(NE) $\quad<$ p $>$ is not true if and only if $<$ not $p>$ is true.

where the if and only if in play in the principle is taken to be a material bicondtional. Consider a case in which $<p>$ is neither true nor false. Then, also $<$ not-p $>$ is neither true nor false. Thus, the right-hand-side of $\mathrm{NE}$ is false. However, it is true that $\langle\mathrm{p}\rangle$ is not true, and thus we have that the left-hand-side of NE is true. Given that IA crucially relies on the validity of NE, there is no argument to begin with. This would make our strategy idle. Call this the Dialectical Worry.

Secondly, a more general worry concerns the validity of ES. Consider the case in which $<p>$ is neither true nor false. Then we have that while the right-hand-side is neither true nor false, the left-hand-side turns out to be false. Renouncing to the general validity of ES would of course be a disastrous result given the central role that it plays in the deflationary conception of truth. Call this the Compatibilism Worry.

Concerning the dialectical worry, a reply is offered by Wright (1992: 63). The aim of IA is preserved even if it is conceded that there are false instances of NE-i.e. those involving gappy propositions. This is because all instances of NE featuring non-gappy propositions hold true and thus, ceteris paribus, the argument for potential extensional divergence between truth and justification goes through. Thus, even in a non-classical framework the dialectical effectiveness of IA is preserved and our reply above still holds.

As for the compatibilism worry, we outline a strategy that could be developed in line with the non-classical strategy. We follow a suggestion made by Greenough (2010) of adopting a threevalued logic similar to K3. Such a logic is the same as K3 except for the conditional which is evaluated as true in the following two scenarios: (i) when both antecedent and consequent are gappy and (ii) when the antecedent is gappy and the consequent false. ${ }^{17}$ The resulting truth-tables for the conditional and the biconditional are the following:

${ }^{17}$ Künne (2003: 351-355) makes a similar move, but he relies on Smiley's adaptation of Bochvar's three-valued logic. 


\begin{tabular}{|c|c|c|c|}
\hline$f \rightarrow$ & 1 & $i$ & 0 \\
\hline 1 & 1 & $i$ & 0 \\
\hline$i$ & 1 & 1 & 1 \\
\hline 0 & 1 & 1 & 1 \\
\hline
\end{tabular}

\begin{tabular}{|c|c|c|c|}
\hline$f \leftrightarrow$ & 1 & $i$ & 0 \\
\hline 1 & 1 & $i$ & 0 \\
\hline$i$ & $i$ & 1 & 1 \\
\hline 0 & 0 & 1 & 1 \\
\hline
\end{tabular}

Let's call this logic GB3. ${ }^{18}$ In GB3 contraposition and reductio fail, but contrary to K3-and in analogy with Lukasiewicz's three-valued logic L3 - there are some logical truths such as "if P, then P'. Crucially, GB3 semantics evaluates every instance of ES as true. In fact, even with gappy propositions, the biconditional:

ES $\quad<p>$ is true if and only if $p$

turns out as true when $<\mathrm{p}>$ is gappy and (consequently) $<\mathrm{Tp}>$ is false. In this respect, the minimal theory of deflationism is safe since the non-paradoxical instances of the equivalence schema turn out true within this logic.

\section{Objections and Replies}

Before concluding, let us deal with a couple of objections that threaten to jeopardise our project. ${ }^{19}$

The first objection goes as follows. Central to our case against Normative Divergence is that a subject's actual acquaintance with (say) a piece of food $\mathbf{f}$ is necessary for a taste-property to be instantiated in $\mathbf{f}$. This seems radical even granted the underlying relativism. If I have six meatballs on a plate and I have eaten the first five and found them tasty, isn't it true that if the physical makeup of the sixth is like that of the first five, the sixth meatball too is such that it is tasty for me?

We believe we have a straightforward reply to this objection. Let's call the sixth meatball $\mathbf{f}^{*}$. Given that $\mathrm{S}$ has had a pleasant gustatory experience at $\mathrm{t}$ of another meatball, $\mathbf{f}$, which belongs to $\mathbf{f}^{*}$ 's homogeneity class we have that, at $\mathrm{t}, \mathbf{f *}$ is tasty relative to the world of taste of S. This holds in virtue of RRD (both classical and non-classical). Moreover, given that by hypothesis $\mathrm{S}$ has a propositional justification to believe that she has had a pleasant experience of $\mathbf{f}$ (via PAGE), and assuming that $\mathrm{S}$ has an a priori justification for $\mathrm{RRD}$, by closure $\mathrm{S}$ has propositional justification to

\footnotetext{
18 Broome (1984) introduced this conditional to address Evans' challenge of possible indeterminate identities. Greenough 2010 discusses this conditional in the context of other gappy logics such as K3 and L3. Hence the label G(greenough)B(roome)3.

19 Thanks to an anonymous referee for making us aware of these concerns.
} 
believe that $\mathbf{f}^{*}$ is tasty. Thus, no divergence between truth and justification is established by this case.

The second objection we would like to address goes as follows. Consider a meatball Max. Here is how it can be true that Max is tasty for me without my being justified in believing so: I ate Max and found it tasty but I have other information which plausibly but misleadingly suggests that it wasn't Max I found tasty. Or vice versa: I haven't tasted Max and Max isn't tasty for me, but I get evidence which makes it rational for me to believe it was Max I tasted, and found tasty. In this way, it seems that truth and justification can come apart, along the lines of Normative Divergence.

One of the principles at the core of our epistemology of basic taste is what we call the primacy of experience thesis according to which the justification for a basic taste judgement consists in and is exhausted by the subject's gustatory responses to her experience. In the first example provided by the objection, given that $\mathrm{S}$ has in fact tasted Max and has had a positive gustatory reaction, according to the primacy of experience principle $S$ has propositional justification for the proposition that Max is tasty. The misleading information that $\mathrm{S}$ acquires has no epistemic effectin particular, no undercutting effect—on S's propositional justification for $<$ Max is tasty $>$. What such information does is to defeat $S$ 's justification for the proposition $<$ S believes that Max is tasty $>$ together with S's ability to claim her justification for $<$ Max is tasty $>$. No misalignment between truth and justification is enforced by the first example. Analogous considerations apply to the second example. This is a case in which, given the primacy of experience thesis, $\mathrm{S}$ has no justification for $<$ Max is tasty $>$ and given RD such a proposition isn't true-whether its negation is true and $\mathrm{S}$ has justification for its negation depends on which model of RD we opt for, but this is not relevant in order to address the objection. The kind of misleading evidence that makes $\mathrm{S}$ believe that it was Max that $\mathrm{S}$ tasted and thus that Max is tasty does not function as propositional justification for $<$ Max is tasty $>$, but, again, as justification for the proposition that $<\mathrm{S}$ believes that Max is tasty $>$. In conclusion, as soon as our epistemology for basic taste judgement is accepted, no misalignment between truth and justification is enforced by the examples presented in the objection. ${ }^{20}$

\section{Conclusions}

Let's conclude by emphasising what we achieved in this paper. We argued that one particular inflationary line of argument against deflationism-i.e. that developed by Wright's IA—can be blocked locally. We developed a metaphysical and epistemological framework for the domain of

\footnotetext{
${ }^{20}$ In Ferrari and Moruzzi (2018) we also consider the case of defeaters (both undermining and overriding) in order to show that they do not break the normative alignment between truth and justification.
} 
basic taste that in virtue of its subjectivist and relativist features invalidates Wright's argument by blocking Normative Divergence.

Two points in relation to the take-home lesson of this project should be highlighted. First, we said nothing about other potential inflationary arguments-e.g., the so-called success argument (Putnam 1978). If the success argument is globally sound, then no purely deflationary truth property would be available. We have reasons to suspect that in virtue of the metaphysical and epistemological framework outlined in this paper for the domain of basic taste, also the success argument is deemed to fail in that domain. But we haven't argued for this here.

However-and this brings us to the second point - even conceding that other arguments may succeed where Wright's IA has locally failed-i.e. in forcing some kind of global inflation on truth's nature- the main point made in this paper remains solid. The point is simply that because IA fails in at least one domain we need to countenance a non-normative truth property. We take this conclusion to be already quite significant for the debate concerning the metaphysics of truthin particular for the alethic pluralism debate. With the exception of Beall's deflationary pluralism (Beall 2013), ${ }^{21}$ all forms of alethic pluralism to date have rejected the possibility of having a nonnormative truth property alongside a plurality of normative truth properties. And this is because, everybody in the debate has taken for granted that Wright's IA is globally sounds-i.e. that it shows that, for every truth apt domain of discourse, there is a potential extensional divergence between truth and justification. Having shown that, given certain metaphysical and epistemological assumptions that seem palatable in the taste domain, a non-normative truth property operating in the domain of taste can be defended, we have opened the possibility of extending the truth pluralist framework in such a way as to include, among a plurality of normative truth properties, also a nonnormative truth property. ${ }^{22,23}$

\footnotetext{
${ }^{21}$ His pluralism_- "Deflated Truth Pluralism"- is entirely deflationary in nature in that it allows for no non-deflationary truth property, and it is motivated by an attempt to avoid semantic paradoxes. Thus, Beall is engaging with a radically different project from that we have undertaken in this paper.

22 We carry this project out in Ferrari and Moruzzi 2018.

23 We would like to thank: Elke Brendel, Massimo Dell'Utri, Douglas Edwards, Patrick Greenough, Eline Busck Gundersen, Thomas Grundmann, Paul Horwich, Nathan Kellen, Michael Lynch, Nikolaj Jang Lee Linding Pedersen, Erik Stei, Elena Tassoni, Joe Ulatowski, Giorgio Volpe, Chase Wrenn, Cory Wright, Jeremy Wyatt, Luca Zanetti, and two anonymous referees from the American Philosophical Quarterly. Special thanks go to Crispin Wright for his extraordinary personal and intellectual encouragement. Ferrari would also like to acknowledge the generous support of the Deutsche Forschungsgemeinschaft (DFG - BR 1978/3-1) for sponsoring his postdoctoral fellowship at the University of Bonn. While working on this paper, both authors have benefitted from participation in the Pluralisms Global Research Network (National Research Foundation of Korea grant no. 2013S1A2A2035514). This support is also gratefully acknowledged.
} 


\section{References}

Beall, JC (2013) "Deflated truth pluralism", in Pedersen \& Wright (2013) (eds.) Truth and Pluralism: Current Debates, Oxford: Oxford University Press: 323-38.

Borghini (2012) “On Being the Same Wine”, Rivista di Estetica 52 (51):175-92.

Broome, J. (1984) "Indefiniteness in Identity", Analysis 44(1):6-12.

Edwards, J. (1997) "Is Tennant selling truth short?", Analysis 57: 152-58.

Edwards, J. (1999) "Prizing Truth from Warranted Assertibility: Reply to Tennant", Analysis 59(4):300-8

Einheuser, I. (2008) “Three forms of Truth Relativism”, in García-Carpintero \& Kölbel (eds.) Relative Truth, Oxford: Oxford University Press:187-203.

Ferrari, F. (2018) “The Value of Minimalist Truth”, Synthese 195: 1103-1125.

Ferrari, F. and Moruzzi, S. (2018) "Ecumenical Alethic Pluralism", Canadian Journal of Philosophy (online first - https://doi.org/10.1080/00455091.2018.1493880).

Fine, K. (2005) Modality and Tense, Oxford: Oxford University Press.

Fine, K. (2012) “Guide to Ground” In Correia, F. \& Schnieder, B. (eds.), Metaphysical Grounding. Cambridge: Cambridge University Press: 37-80.

Greenough, P. (2010) “Deflationism and Truth-Value Gaps”, In Nikolaj Pedersen \& Cory D.

Wright (eds.), New W aves in Truth. Palgrave-Macmillan.

Horwich, P. (1998) Truth, 2nd ed., Oxford: Oxford University Press.

Horwich, P. (2018) “Is Truth a Normative Concept?", Synthese 195: 1127-1138.

Jackson, A. (2016) "From relative truth to Finean non-factualism", Synthese, 193 (3): 971-98.

Künne, W. (2003) Conceptions of Truth, Oxford: Oxford University Press.

Lynch, M. (2006) "ReWrighting Pluralism", The Monist 89(1): 63-84.

Miller, A. (2001) “On Wright's Argument Against Deflationism”, Philosophical Quarterly 51:527-31.

Price, H. (1998) “Three Norms of Assertibility, or How the Moa became Extinct”, Nô̂s 32(12): 24154.

Priest, G. (2001) Introduction to Non-Classical Logic, Cambridge: Cambridge University Press.

Putnam, H. (1978) Meaning and the Moral Sciences, London: Routledge and Kegan Paul.

Robson, J. (2013) “Appreciating the Acquaintance Principle: A Reply to Konigsberg”, British Journal of Aesthetics 53(2):237-245.

Rovane, C. (2013) The Metaphysics and Ethics of Relativism, Cambridge(MA): Harvard University Press.

Rumfitt, I. (1995) “Truth Wronged: Crispin Wright's Truth and Objectivity”, Ratio 8:100-7.

Spencer, J. (2016) "Relativity and Degrees of Relationality", Philosophy and Phenomenological Research 42(2): 432-459.

Tennant, N. (1995) “On negation, truth and warranted assertibility”, Analysis 55: 98-104.

Tennant, N. (1998) "The full price of truth”, Analysis 58:221-28.

Williamson, T. (1997) "Imagination, Stipulation and Vagueness", Philosophical Issues 8: 215-228. 
Williamson, T. (2000) Knowledge and Its Limits, Oxford: Oxford University Press.

Wollheim, R. (1980) Arts and its Object, Cambridge: University Press.

Wrenn, C. (2015) Truth, Cambridge: Polity.

Wright, C. (1992) Truth and Objectivity, Cambridge(MA): Harvard University Press.

Wright, C. (1998) “Truth: A Traditional Debate Reviewed”, Canadian Journal of Philosophy, SV:31-74.

Wyatt, J. (2016) “The many (yet few) faces of deflationism”, Philosophical Quarterly 66(263): 362-82. 